\title{
Magnetoresistance oscillations in La-based metallic glass
}

\author{
WANG YongTian ${ }^{1,2 *}$, LIU ZongDe ${ }^{1,3} \&$ WANG WeiHua ${ }^{2}$ \\ ${ }^{1}$ Key Laboratory of Condition Monitoring and Control for Power Plant Equipment of Ministry of Education, North China Electric Power Univer- \\ sity, Beijing 102206, China; \\ ${ }^{2}$ Institute of Physics, Chinese Academy of Sciences, Beijing 100190, China; \\ ${ }^{3}$ Suzhou Research Institute of North China Electric Power University, Suzhou 215123, China
}

Received April 13, 2011; accepted June 28, 2011

\begin{abstract}
We report that $\mathrm{La}_{60} \mathrm{Fe}_{30} \mathrm{Al}_{10}$ metallic glass has clear, reproducible, periodic variation in its differential resistance as a function of a perpendicular magnetic field below its superconducting transition temperature. The oscillation period corresponds to a superconducting flux quantum. The observed phenomena originate from the Little-Parks-like resistance oscillations in the cylindrical La nanorod with a high aspect ratio and uniform orientation precipitated on the ribbon surface. The highly-oriented La nanocrystals prepared on a flexible glass substrate offer an opportunity for integrating numerous superconducting circuits into a single chip.
\end{abstract}

metallic glass, superconducting, resistance oscillation

Citation: Wang Y T, Liu Z D, Wang W H. Magnetoresistance oscillations in La-based metallic glass. Chinese Sci Bull, 2011, 56: 3984-3987, doi: 10.1007/ s11434-011-4764-9

The magnetoresistance oscillations of low-dimensional superconductors, such as nanowire, nanobelt, or granular systems, have been extensively studied [1,2]. This is one of the most intriguing problems in condensed-matter physics, and this technology is widely used to develop new kinds of superconducting quantum interference devices (SQUIDs), such as SQUIDs based on carbon nanotubes [3] and SQUIDs made via the DNA templating of superconducting nanowires [4]. However, the low-dimensional samples in these studies are either single or quasi-1D. An oriented 2D nanoarray with a large aspect ratio and flexible substrate is highly desirable.

In this letter, we report the fabrication of a simple and flexible superconducting device based on a La-based metallic glass. Below the superconducting transition temperature, $T_{\mathrm{c}}$, the ribbon, which has high intrinsic flexibility and strength, exhibited a clear periodic modulation in its differential resistance as a function of a perpendicular magnetic field. The oscillations resulted from a two-dimensional

*Corresponding author (email: wyt@ncepu.edu.cn) array composed of La nanocrystals with high aspect ratios and uniform orientations. These arrays spontaneously assembled on the free surface of the glass ribbon upon quenching, which is not the case for other nanoparticles and nanowires [5-7]. The work presents an advanced application of the device for an implementation using metallic glasses as the precursors for the nano-material [8-11].

\section{Experimental}

The $\mathrm{La}_{60} \mathrm{Fe}_{30} \mathrm{Al}_{10}$ ribbons were prepared using a single roller melt-spinning apparatus at $5-25 \mathrm{~m} / \mathrm{s}$ in an argon atmosphere. The structure was characterized via X-ray diffraction (XRD) in a MAC M03 XHF diffractometer with $\mathrm{CuK} \alpha$ radiation. Thermal analysis was performed using a Perkin-Elmer DSC-7 differential scanning calorimeter (DSC). The topography of the ribbon was studied using an atomic force microscope (AFM, Digital Instruments NanoScope IIIa D-3000 AFM). The magnetism and standard four-point electrical resistance measurements were performed using a PPMS 6000. 


\section{Results}

Figure 1 shows the XRD patterns of the $\mathrm{La}_{60} \mathrm{Fe}_{30} \mathrm{Al}_{10}$ ribbons for various wheel velocities. For the ribbon spun at 25 $\mathrm{m} / \mathrm{s}$, the XRD pattern of the bottom surface (in contact with the copper roller) shows a broad maximum peak indicating an almost amorphous phase. The sharp crystallization peak of the inset DSC curve confirms its glass-like nature. No amorphous phase can be detected from the ribbon free surface, but a strong and broadening crystalline peak is superimposed on the XRD curve. When the spin velocity decreases to 10 and $5 \mathrm{~m} / \mathrm{s}$, the intensity of the peak decreases. Through closer examination of the XRD pattern, one can find a much stronger $\mathrm{La}(002)$ peak, which suggests that the hexagonal La phase has a well-defined texture with the $c$-axis predominantly perpendicular to the ribbon plane [12]. According to the Scherrer formula [13,14], the peak is broadened because of the formation of ultrafine nanocrystals.

Figure 2(a)-(d) shows the AFM micrographs of the $\mathrm{La}_{60} \mathrm{Fe}_{30} \mathrm{Al}_{10}$ ribbon spun at $5 \mathrm{~m} / \mathrm{s}$ (left) and $25 \mathrm{~m} / \mathrm{s}$ (right). The bottom shows the corresponding cross-section analysis $(1 \mu \mathrm{m} \times 1 \mu \mathrm{m})$. For the ribbon at $5 \mathrm{~m} / \mathrm{s}$, some nanocrystals with random distribution were observed on the free surface, and the size and distribution of the nanocrystals were far from uniform. However, when the wheel velocity was increased to $25 \mathrm{~m} / \mathrm{s}$, a large number of columelliform nanocrystals with a lateral width of $\sim 100 \mathrm{~nm}$ were assembled into a compact 2D film over the whole ribbon free surface. The improved texture also resulted in the greatly enhanced intensity of the La (002) peak. The precipitation of Rare-Earth (RE) particles is a common phenomenon for the RE-Fe-Al glass-forming system [11,12]. The La nanocrystals on the surface have a stronger temperature gradient in

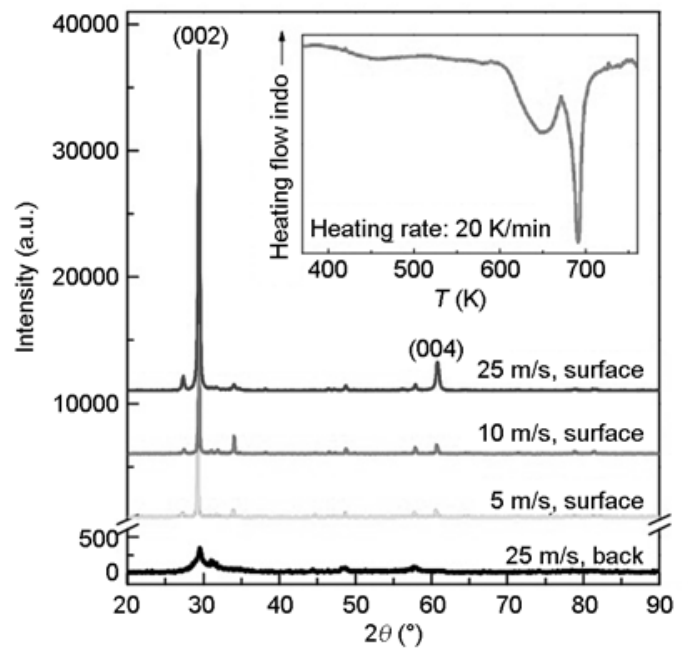

Figure 1 XRD patterns for the $\mathrm{La}_{60} \mathrm{Fe}_{30} \mathrm{Al}_{10}$ ribbons at various wheel velocities. The inset shows the DSC curve of the ribbons at $25 \mathrm{~m} / \mathrm{s}$. The heating rate is $20 \mathrm{~K} / \mathrm{min}$.
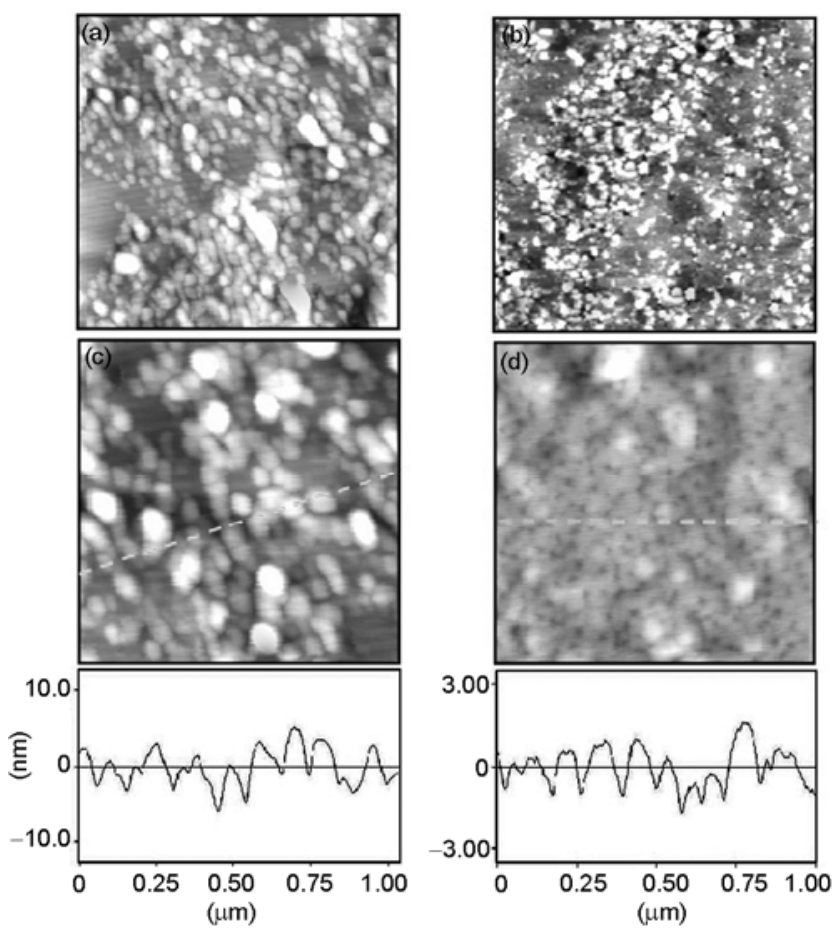

Figure 2 Topographic AFM images of the $\mathrm{La}_{60} \mathrm{Fe}_{30} \mathrm{Al}_{10}$ ribbon free surface and corresponding cross-section analysis. (a) $5 \mathrm{~m} / \mathrm{s}, 2 \mu \mathrm{m} \times 2 \mu \mathrm{m}$; (b) $25 \mathrm{~m} / \mathrm{s}, 10 \mu \mathrm{m} \times 10 \mu \mathrm{m}$; (c) $5 \mathrm{~m} / \mathrm{s}, 1 \mu \mathrm{m} \times 1 \mu \mathrm{m}$; (d) $25 \mathrm{~m} / \mathrm{s}, 1 \mu \mathrm{m} \times 1 \mu \mathrm{m}$.

the direction perpendicular to the ribbon plane [11]. Therefore, these La nuclei tend to grow normal to the ribbon plane, and finally assemble into the film.

Figure 3 shows the relative resistance, $R / R_{10 \mathrm{~K}}$, versus the temperature, $T$, of the ribbon in different magnetic fields. The left inset shows the standard four-probe setup for ribbon in-plane and transverse resistance measurements $\left(R_{\mathrm{S}}\right.$ and $R_{\mathrm{T}}$ ). The $R_{\mathrm{T}}$ is 10 times less than the in-plane resistivity $R_{\mathrm{s}}$. Both $R_{\mathrm{T}}$ and $R_{\mathrm{s}}$ show metallic behaviors from room temperature to $T_{\mathrm{c}}$, with residual resistance ratios, $R(300$ $\mathrm{K}) / R(10 \mathrm{~K})$, of 3.2 and 3.5 , respectively. This indicates that direct ohmic contact is established between the deposited $\mathrm{Ag}$ electrode and ribbon [15]. At $H=0 \mathrm{~T}$, the $R$ shows a sharp drop near $5 \mathrm{~K}$ before decaying to zero, indicating the ribbon is entering a superconducting state. The initial temperature of superconductivity is near the reported hexagonal $\alpha$-La polycrystalline with $T_{\mathrm{c}}=4.9 \mathrm{~K}$ [16]. The transition range is broad, $\Delta T \approx 2 \mathrm{~K}$, which is significantly greater than that in similarly prepared bulk La-based alloys. This indicates we are approaching the one dimensional limit, which is typical for a 1D superconductor because of its susceptibility to thermal fluctuation $[4,5]$. The right inset of Figure 3 shows the $H$ dependence of the resistance with $H$ perpendicular to the ribbonfree surface at 2,4 , and $6 \mathrm{~K}$, respectively. Above $T_{\mathrm{c}} \approx 5 \mathrm{~K}$, both $R_{\mathrm{s}}$ and $R_{\mathrm{T}}$ remain constant when the magnetic field increases, whereas below $T_{\mathrm{c}}$ the destruction of the superconducting state by the magnetic field at 2 and $4 \mathrm{~K}$ results in different stages with slight slope 


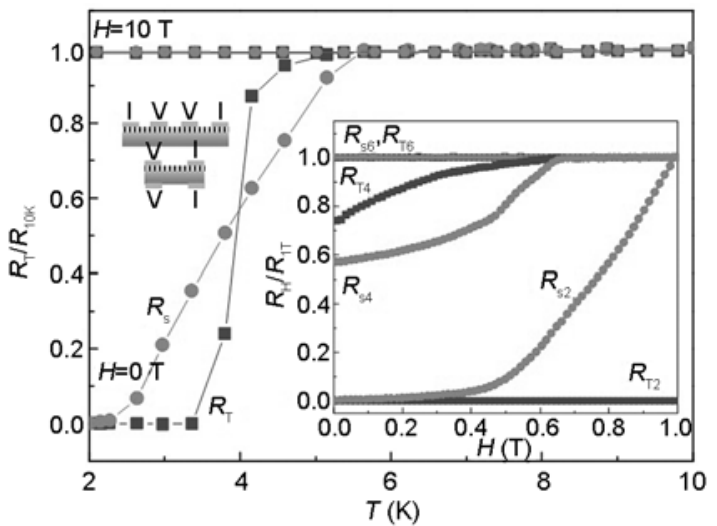

Figure 3 The relative resistance, $R / R_{10 \mathrm{~K}}$, versus temperature measurements of the ribbon spun at $25 \mathrm{~m} / \mathrm{s}$. The left inset diagram shows the standard four-probe setup for surface and transverse resistance measurements $\left(R_{\mathrm{S}}\right.$ and $\left.R_{\mathrm{T}}\right)$, respectively. The four orange regions denote the silver plate electrodes. The right inset shows the resistance $R_{\mathrm{s}}$ and $R_{\mathrm{T}}$ versus magnetic field measurements at 2,4 , and $6 \mathrm{~K}$, respectively.

changes until the critical field.

An analysis of the differential magnetoresistance $\mathrm{d} R / \mathrm{d} H$ with respect to $H$ is shown in Figure 4. Note that differentiating the curve to magnify the slight slope change is a powerful and widely-used data analysis method [5]. This figure shows that, for ribbons spun at $5 \mathrm{~m} / \mathrm{s}$, the $\mathrm{d} R / \mathrm{d} H$ includes three distinct regimes, which are the same as those of the superconducting granular film with disordered grains [17] No oscillations are observed. However, for ribbons at 25 $\mathrm{m} / \mathrm{s}, \mathrm{d} R / \mathrm{d} H$ has well-defined oscillations. The oscillation period $\Delta H=0.36 \mathrm{~T}$ is reproducible and independent of temperature and the magnetic history of the ribbon, i.e., sweeping the magnetic field up or down. Although it fluctuates greatly, the amplitude of the oscillations does not show a systematic dependence on $H$ below the critical temperature $T_{\mathrm{c}}$, above which significant oscillations are no longer detected.

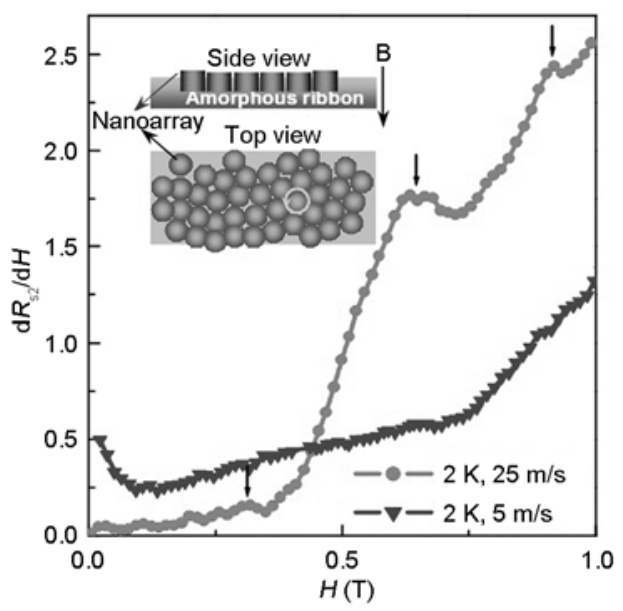

Figure 4 The derivative $\mathrm{d} R_{\S} / \mathrm{d} H$ vs. $H$ at $2 \mathrm{~K}$ for the ribbons spun at 5 and $25 \mathrm{~m} / \mathrm{s}$, respectively. The inset shows the diagram of La-based glass ribbon. The circle denotes the superconducting screening current loops.

\section{Discussion}

The resistance oscillations in conventional SQUIDs and Little-Parks (LP) resistance oscillations in thin-walled cylinders are the most well-known examples of quantum interference [4]. Taking into account the circular La nanorods and $T$-independent period, it seems that there are mesoscopic superconducting ring-like structures in the ribbon surface, which may induce the periodic magnetoresistance oscillation in the superconducting regime, such as Little-Parks-like oscillations. Our findings deviate significantly from the expected outcome because the standard LP oscillations are expected in a superconducting hollow cylinder near $T_{\mathrm{c}}$. This deviation may occur because La nanorods with a high aspect ratio are not fully superconducting. Even for $H=0 \mathrm{~T}$, the core of the nanorod shows a finite resistance below $2 \mathrm{~K}$. The broadening and the finite resistance below the onset of superconducting transition are likely the consequence of the size confinement in 1D created by the thermally-activated phase slips near $T_{\mathrm{c}}$ [2] or the quantum phase slips far below $T_{\mathrm{c}}$ [2]. As the magnetic field increases, even at temperatures well below $T_{\mathrm{c}}$, there is a superconducting cylindrical shell surrounding the $\mathrm{La}$ nanorod. In the superconducting-dominated states, the magnetic field creates Meissner screening currents and through them suppresses the energy barrier for the phase slips. The net current is a combination of normal and super currents. The periodic oscillations are produced by the latter, which likely reflected the oscillations of the screening currents around the La nanorod as a function of the magnetic field.

The observed magnetoresistance oscillation is currently not well understood. We suggest the following quantitative explanation for LP oscillations [18], in which the period of the oscillations, $\Delta H$, is controlled by the superconducting flux quantum $\Phi_{0}=h / 2 e$ (here $e$ is the electron charge and $h$ is Plank's constant), divided by the cross-sectional area $S$ enclosing the magnetic field, $\Delta H \cdot S=\Phi_{0}=2.07 \times 10^{-15} \mathrm{~Wb}$, for a superconducting circle loop or cylinder. The sum of the externally-applied magnetic flux $\Phi$ and the flux induced by the superconducting screening currents surrounding the La nanorods is quantized in units of the flux quantum $\Phi_{0}$. The screening currents are absent when $\Phi=n \Phi_{0}$, but the screening currents reach their maximum amplitude when $\Phi=(n+1 / 2) \Phi_{0}$, resulting in a minimum value of $T_{\mathrm{c}}$ for the higher flux values. Experimentally, the LP oscillations of $T_{\mathrm{c}}$ can be observed as a periodic variation of the $R$ with $H$ at a fixed temperature. Therefore, the $\mathrm{d} R / \mathrm{d} H$ is characterized by the regular appearance of minima or maxima in $H_{\mathrm{n}}$. The measured period, $\Delta H=0.36 \mathrm{~T}$, theoretically corresponds to a superconducting current loop with $R_{1}=43 \mathrm{~nm}$, which is in good agreement with the La nanorod experimental radius (the green circle, $40-50 \mathrm{~nm}$ ) measured via AFM (see the inset of Figure 4). Thus, the oscillation periods are attribut- 
ed to the superconducting screening currents surrounding the La nanorods, i.e. the dominant cell area in the La-based array.

Note that although the oscillation is reproducible for different samples, the period of oscillation is not well defined. To further test the prediction, we compare the La nanoarray with a superconducting granular Sn film and wire [17]. Because the $2 \mathrm{D}$ film is macroscopic, and there is a wide distribution of loop size, the oscillation of the superconducting screening currents will average out and no oscillations will be detected. This is similar to the case for ribbons with disordered La nanocrystals (spun at $5 \mathrm{~m} / \mathrm{s}$ ). However, the 1D granular Sn wire with reduced dimensionality begins to show a series of periods [17]. Analogously, the oriented La nanoarray originated from the ribbon spun at $25 \mathrm{~m} / \mathrm{s}$, its degree-of-freedom is even less than that in the ribbon at 5 $\mathrm{m} / \mathrm{s}$. Therefore, there is only one dominant superconducting loop around the La nanorod, which is responsible for the main oscillation periods.

\section{Conclusion}

In summary, a La nanoarray with a large aspect ratio and uniform orientation was assembled directly from a $\mathrm{La}_{60} \mathrm{Fe}_{30} \mathrm{Al}_{10}$ glass alloy upon quenching. This nanoarray is similar in function to a simple and flexible SQUID-like device. The present single-step formation method eliminates the conventional cumbersome process and offers an easy way to fabricate advanced functional materials using metallic glass as precursors.

This work was supported by the National Natural Science Foundation of China (51101056, 50731008 and 50921091), the National Basic Research Program of China (2007CB613904), the Fundamental Research Funds for the Central Universities (09Q44), and the State Key Lab of Advanced Metals and Materials (2010Z-02).
1 Wang J, Shi C T, Tian M L, et al. Proximity-induced superconductivity in nanowires: Minigap state and differential magnetoresistance oscillations. Phys Rev Lett, 2009, 102: 247003

2 Tian M L, Wang J, Zhang Q, et al. Superconductivity and quantum oscillations in crystalline Bi nanowire. Nano Lett, 2009, 9: 3196-3202

3 Cleuziou J P, Wernsdorfer W, Bouchiat V, et al. Carbon nanotube superconducting quantum interference device. Nat Nanotech, 2006, 1: 53-59

4 Hopkins D S, Pekker D, Golbart P M, et al. Quantum interference device made by DNA templating of superconducting nanowires. Science, 2005, 308: 1762-1765

5 Johansson A, Sambandamurthy G, Shahar D. Nanowire acting as a superconducting quantum interference device. Phys Rev Lett, 2005, 95: 116805

6 Hasselbach K, Mailly D, Kirtley J R. Micro-superconducting quantum interference device characteristics. J Appl Phys, 2002, 91: 4432

$7 \mathrm{Xu}$ Q B, Castillejos R P, Li Z F, et al. Fabrication of high-aspect-ratio metallic nanostructures using nanoskiving. Nano Lett, 2006, 6: 2163-2165

8 Inoue A, Shimizu T, Yamaura S, et al. Development of glassy alloy separators for a proton exchange membrane fuel cell (PEMFC). Mater Trans, 2005, 46: 1706-1710

9 Schroers J, Lohwongwatana B, Johnson W L, et al. Gold based bulk metallic glass. Appl Phys Lett, 2005, 87: 061912

10 Kim J J, Choi Y, Suresh S, et al. Nanocrystallization during nanoindentation of a bulk amorphous metal alloy at room temperature. Science, 2002, 295: 651-657

11 Wang Y T, Bai H Y, Pan M X, et al. Giant enhancement of magnetocaloric effect in metallic glass matrix composite. Sci China Ser G-Phys Mech Astron, 2008, 51: 337-348

12 Sun Z G, Löser W, Eckert J, et al. Phase separation in $\mathrm{Nd}_{60-x} \mathrm{Y}_{x^{-}}$ $\mathrm{Fe}_{30} \mathrm{Al}_{10}$ melt-spun ribbons. Appl Phys Lett, 2002, 80: 772

13 Guo W H, Kui H W. Bulk nanostructured alloy formation with controllable grain size. Acta Mater, 2004, 8: 2117-2121

14 Wang W H, Dong C, Shek C H. Bulk metallic glasses. Mater Sci Eng R, 2004, 44: 45-89

15 Stankovich S, Dikin D A, Dommett G H B, et al. Graphene-based composite materials. Nature, 2006, 442: 282-286

16 Agyeman K, Muller R, Tsuer C C. Alloying effect on superconductivity in amorphous lanthanum-based alloys. Phys Rev B, 1979, 19: 193-198

17 Herzog A V, Xiong P, Dynes R C. Magnetoresistance oscillations in granular Sn wires near the superconductor-insulator transition. Phys Rev B, 1998, 58: 14199-14202

18 Vloeberghs H, Moshchalkov V V, Haesendonck C V, et al. Anomalous Little-Parks oscillations in mesoscopic loops. Phys Rev Lett, 1992, 69: 1268-1271

Open Access This article is distributed under the terms of the Creative Commons Attribution License which permits any use, distribution, and reproduction in any medium, provided the original author(s) and source are credited. 